\title{
Design and Parametric Analysis of GaN on Silicon High Electron Mobility Transistor for RF Performance Enhancement
}

Jeetendra Singh ( $\sim$ jeetendras15.phd@nitj.ac.in )

NIT Sikkim: National Institute of Technology Sikkim

Archana Verma

Rajkiya Engineering College Kannauj

Vijay Kumar Tewari

Rajkiya Engineering College Kannauj

Shailendra Singh

PSIT: Pranveer Singh Institute of Technology

\section{Research Article}

Keywords: GaN on Silicon, high electron mobility transistor, gate work-function, doping effects, 2 dimensional electron gas

Posted Date: September 3rd, 2021

DOI: https://doi.org/10.21203/rs.3.rs-865726/v1

License: (9) This work is licensed under a Creative Commons Attribution 4.0 International License. Read Full License 


\title{
Design and Parametric Analysis of GaN on Silicon High Electron Mobility Transistor for RF Performance Enhancement
}

\author{
Jeetendra Singh*1(jeetendras15.phd@nitj.ac.in), Archana Verma², Vijay Kumar Tewari ${ }^{3}$ and Shailendra Singh ${ }^{4}$ \\ 1.- ECE, NIT Sikkim, 2. - EL, REC Kannauj, 3. - EE, REC Kannauj, 4. - ECE, PSIT Kanpur
}

\begin{abstract}
The need of performance enhancement at the RF and millimeter wave is highly desirable to eliminate the heating effects and power dissipation. Silicon substarte found to be a suitable candidate which reduces about $70 \%$ channel temperature than sapphire. To achieve high performance, a GaN on the Silicon substrate high electron mobility transistor is designed and its various performance paramters are analyzed by varying the design specifications. Moreover, the problem of blocking voltage improvement is resolved by epitaxial design approach. Since, threshold voltage, doping-level, work-function of gate material and channel length are considered as some of the important parameters while device modeling. Therefore, the impact of these parameters is examined and analyzed to enehace the performance and reliability of the device for $\mathrm{RF}$ applications. The performance parameters like trans-conductance, drain current curves are plotted at different state of device physical and electrical parameters. Results exhibits maximum value of transconductance $\mathrm{g}_{\mathrm{m}}=13$ milli-mho, minimum gate capacitance $\mathrm{C}_{\mathrm{g}}=0.5 \mathrm{pf}$, wheras $\mathrm{V}_{\text {th }}$ is varied between -0.25 volts to 0.25 volts.
\end{abstract}

Keywords - GaN on Silicon, high electron mobility transistor, gate work-function, doping effects, 2 dimensional electron gas.

\section{INTRODUCTION}

With the continue down scaling of the transistor leads to switching speed and its efficiency losses in terms of short channel effects and hence renders large power dissipation. Form the past two decades, all the device are reliable on the silicon material for power device, however the silicon has some limits on handling high power devices [1-2]. In some domains, the power electronic devices are reaching to its fundamental limits, where the design and manufacturing unit facing the challenge in terms of operating voltage, temperature and frequency [3-5]. As alternatives of sapphire substarte, researchers move to Silicon substrate for wide bandgap materials such as $\mathrm{GaN}$ or $\mathrm{SiC}$. The related semiconductor like $\mathrm{GaN} / \mathrm{AlGaN} / \mathrm{InGaN}$ on Silicon substrate have been continuously recorded as a promising device for the next generation of high frequency, high electric field with wide temperature range [6-8]. In particular, hetero-structure base on wide band gap materials like $\mathrm{AlGaN} / \mathrm{GaN}$ are preferred in the channel interface to achieve the high mobilty of the carriers. This happens due to its distinctive characteristics due to wide band gap, which yields high breakdown field with high drain current [9-11]. The high electron mobility transistor device commonly used for RF applications likewise in radar, cellular telecommunication, radio astronomy and the satellite broadcasting. These applications employ the low noise with high frequency and high critical electric field [12-14]. The high electron mobility transistor exhibits the three times higher power density than MOSFET which over all reduces the chip area in terms of their output performance. One of the special characteristics of the high electron mobility transistor is the formation of the two-dimensional electron gas molecule where the carrier mobility is extremely high [15-16]. The important feature of $\mathrm{AlGaN} / \mathrm{GaN}$ based high electron mobility transistor structure is basically the formation of electrons confinement at the interface to from two dimensional electron gas (2DEG). The conventional AlGaN/GaN hetero-structure suffers from high $\mathrm{R}_{\mathrm{ON}}$ which owns the 2DEG density in gate-source and gatedrain region, however low $\mathrm{R}_{\mathrm{ON}}$ can be achieved by polarizationinduced high density and 2DEG mobility at the interface. Many other optimal approaches used for lowering the $\mathrm{R}_{\mathrm{ON}}$, like 2DEG depletion can be attained by using a thin AlGaN barrier layer with low Aluminum concentration [17]. The 2DEG cannot be easily- depleted at zero bias in schottky gate contact [18-19] but due to the band-gap, the critical field strength is inversely proportion to the drift region thickness and hence, the ON-state resistance is lower [20].

In this paper, the response of the Gallium nitride $(\mathrm{GaN})$ on Silicon sunbstrate high electron mobility transistor is desined and being analyzed for the high voltage, high switching power for regulation of RF application at high temperature. The operation is associated with efficiently power switching in which the OFF-state voltage is highly blocked with minimum leakage current and $\mathrm{ON}$ state resistance. In order to operate at high voltage, these devices can be limited by the chain factor like subthreshold voltage and device scaling. However, the blocking voltage improvement is a complex problem which can be resolved by, epitaxial design, device structure and its scaling with favorable fabrication technology.

\section{DEVICE STRUCTURE AND SIMULATION FRAMEWORK}

In high electron mobility transistor, the presence of a conduction band offset at the interface of two different bandgap materials produces two-dimensional electron gas (2DEG). The potential well is formed on the material side of the lower bandgap. Over the GaN side of the device, 2DEG is generated when higher bandgap material $(\mathrm{AlGaN})$ develops on lower bandgap material $(\mathrm{GaN})$. Due to ease of growth in the of $\mathrm{GaN}$ over silicon substrate with low cost and large size will make its choice better over the $\mathrm{SiC}$ and other material. Additionally, in heterostructure, GaN employed with polarization field in which two different layers of different bandgap are come together for 
single growth. However, this will create a difference in electric field and surface potential at heterojunction. Figure 1 show the schematic structure of $\mathrm{AlGaN} / \mathrm{GaN}$ in which $\mathrm{AlGaN}$ is common in between source and drain electrodes. The doped high electron mobility transistor structure with $\mathrm{AlGaN}$ doped layer will help in improving the polarization across the layer. This enhances the transport characteristics by imprisonment of electron within the channel.

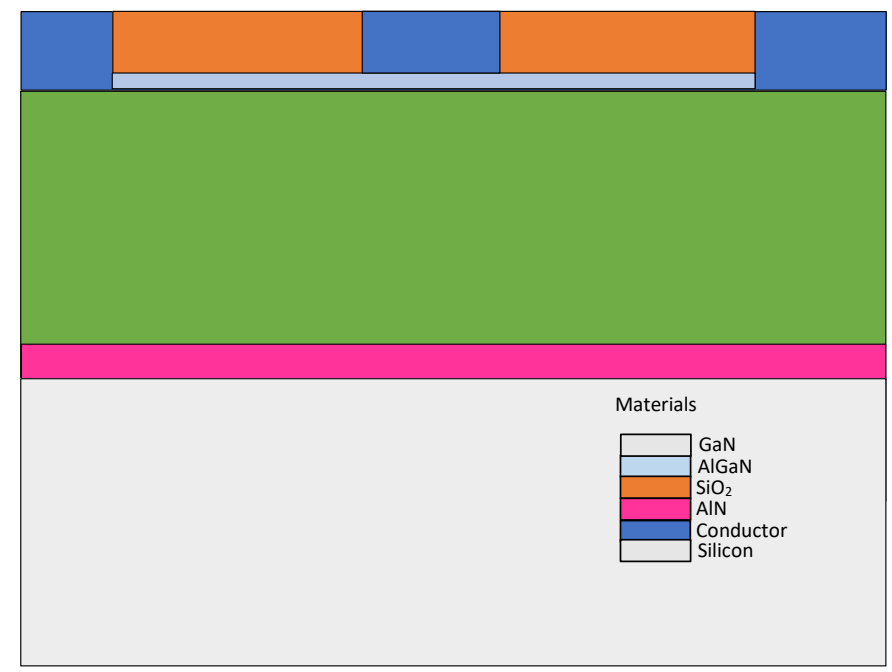

Fig. 1. Schematic diagram of GaN on Silicon substarte high electron mobility transistor (high electron mobility transistor) TCAD tool structure

If the induced charge is positive, electrons will tend to compensate the induced charge resulting in the formation of the channel. The channel electrons in the high electron mobility transistor are referred to as a Two-Dimensional Electron Gas because they are trapped in a quantum well in a very restricted spatial region at the hetero-interface (2DEG) [22]. The equivalent device design parameters of high electron mobility transistor used during simulation work are shown in Table I.

Table I: Device specification of the proposed device $\mathrm{AlGaN} / \mathrm{GaN}$ based high electron mobility transistor.

\begin{tabular}{|c|c|c|}
\hline Parameter Used & Length & Width \\
\hline Device & $6 \mu \mathrm{m}$ & $3 \mu \mathrm{m}$ \\
\hline Channel & $3 \mu \mathrm{m}$ & $0.03 \mu \mathrm{m}$ \\
\hline AlN 2DEG & $6 \mu \mathrm{m}$ & $0.05 \mu \mathrm{m}$ \\
\hline Electrode Gate & $1 \mu \mathrm{m}$ & $0.05 \mu \mathrm{m}$ \\
\hline Electrode Drain & $1 \mu \mathrm{m}$ & $0.035 \mu \mathrm{m}$ \\
\hline Electrode Source & $1 \mu \mathrm{m}$ & $0.035 \mu \mathrm{m}$ \\
\hline Silicon Substrate & $400 \mu \mathrm{m}$ & $0.095 \mu \mathrm{m}$ \\
\hline
\end{tabular}

The simulation of the device is excuted using SilvacoATLAS tool [21]. Drift-deffusion model which is based on the Boltzmann's transport theory along with two advance transport models namely hydrodynamic transport model and energy balance transport model are considered while simulation to sustain the carrier temperature instead of the local electric field. Moreover, polarization model of GaN, fermi-dirac model, SRH model, low and high electric field models, and Selberherr's impact ionization model are included during the simualtiing the device structure. A detailed analysis of the proposed device of $\mathrm{AlGaN} / \mathrm{GaN}$ high electron mobility transistor is given with the structure dimensions. More attention towards thermal conduction and Schottky-gate reverse bias tunneling are carried out. The affecting parameters like doping concentration, channel length, gate work-function, trans-conductance and capacitance characteristics are observed by varying $\mathrm{V}_{\mathrm{GS}}$.

\section{A. Polarization Effect}

$\mathrm{AlGaN} / \mathrm{GaN}$ have special characteristics which holds the two-dimensional electron gas without doping process. This happens due to piezoelectric properties and spontaneous polarization which is found in crystal structure of III-nitrides and can be represent by Figure 2. The high tendency of atom to attract an electron bonding which is of tetrahedrally structure between III-nitrides elements, results in spontaneous polarization [23-24]. In the crystal structure, the epitaxial GaN layer was grown at normal to (0001) position. Due to lack of inversion symmetry, it will result in spontaneous polarization in $\langle 0001\rangle$ direction.

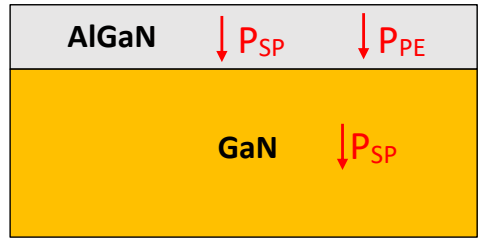

Fig. 2. Schematic diagram representing the spontaneous effect with piezoelectric polarization of $\mathrm{AlGaN} / \mathrm{GaN}$ high electron mobility transfer (high electron mobility transistor) TCAD tool structure.

In comparison to $\mathrm{GaN}$, the $\mathrm{AlGaN}$ is very thin in $\mathrm{AlGaN} / \mathrm{GaN}$ in high electron mobility transistor structure, this is done due to lattice mismatch which need to be equal and constant. The piezoelectric polarization of the interface field can be expressed as:

$$
P_{P E}=2 \varepsilon_{a}\left(e_{31}-\frac{e_{33} c_{13}}{c_{33}}\right)
$$

The above equation is directly proportional to lattice constant $\left(\varepsilon_{a}\right)$ and piezoelectric coefficient of $\mathrm{AlGaN} / \mathrm{GaN}$ in high electron mobility transistor structure. The average polarization effect caused by $\mathrm{AlGaN} / \mathrm{GaN}$, where $\mathrm{GaN}$ consist negative polarization field and $\mathrm{AlGaN}$ consist of positive spontaneous and piezoelectric polarization effect $\left(\mathrm{P}_{\mathrm{SP}}\right)$ [25-26]. Since the spontaneous polarization $\left(\mathrm{P}_{\mathrm{PE}}\right)$ is greater in comparison to the $\mathrm{GaN}$, at the $\mathrm{AlGaN} / \mathrm{GaN}$ interface charges.

$$
\sigma=\left(P_{S P, A l G a N}+P_{P E, A l G a N}\right)-P_{S P, G a N}
$$

Both the equation (1) \& (2), will conclude as a fixed permanent creation of a 2 DEG polarization.

\section{B. Parasitic Effects}

The parasitic effects are also known as trapping effects, as the trap charges (electron and holes) are lie between the channel and the gate contact. The effect induces due to generation of available energy levels in energy bandgap of semiconductor, which are generally related to the lattice mismatched of different materials connected to each other [27]. In the ON condition of the device, it limits the active charges with respect 
to time and stress, which futher causes an effect to the static and dynamic performance of the device. In order to eliminates these trap charges, we need to apply operative electric field. This collision of trap and non-trap charge will make device performance degrading [28].The two main parasitics effects are kink and current collapse effect. The kink effect are more effective at low drain voltage at degrade the $\mathrm{ON}$ drain current performace. However, this can be overcome by the increasing the voltage $\mathrm{V}_{\mathrm{ds}}$ (drain-source voltage), resulting the pinch off voltage to be shifted towards more negative voltages and which implusively raise the drain current. The disturbed DC current will effect on $\mathrm{ON}$ resistance $\left(\mathrm{R}_{\mathrm{on}}\right)$ and transconductance $\left(\mathrm{g}_{\mathrm{m}}\right)$. The upper trap charges of bandgap are acceptor and lower trap charges are donor. The position of the trap charges will play a vital role in the device performance. They are filled with the narrow AlGaN layer, in order to kept the trap charges level below the Fermi level. Due to narrow barrier, the charges will trap and pused hardly by the strong polarization effect of AlGaN. The emmison rate relation will the $V_{D}$ can be exterated by using three given three equations (3), (4)and (5). Initally consider the emission rate to be calculated by Arrhenius equation at random temperature [29].

$$
e=A T^{2} \exp \left(-\frac{E_{F}}{k T}\right)
$$

Where $\mathrm{T}, \mathrm{k}$ and $\mathrm{E}_{\mathrm{F}}$ are temperature, Boltzmann constant and Fermi energy level. The barrier $(\beta)$ decreases due to high electric field with respect to square root of applied electric field given by equation (4).

$$
\Delta \eta \phi_{P F}=\left(\frac{q^{3}}{\pi}\right)^{1 / 2} \sqrt{F}=\beta \sqrt{F}
$$

Here, $\mathrm{q}$ is a electron charges and $\varepsilon$ represent the dielectric constant. The ionization energy of the field can be represented by:

$$
E_{i}(F)=E_{i}(0)-\beta \sqrt{F}
$$

The $\Delta E_{C}$ is energy band gap difference between $\mathrm{AlGaN}$ and $\mathrm{GaN}$, where at interface of $\mathrm{AlGaN}$ and $\mathrm{GaN}, E_{i}(F)$ is difference conduction band and fermi level. The current collapse near drain region occur near the surface of $\mathrm{AlGaN}$ layer resulting the channel depletion.

Fig. 3 is given below to understand energy band diagram of the high electron mobility transistor, there is given three different regions of Metal, AlGaN and GaN. Quantum tunneling valley happens in conduction band, basically the reason behind that being the tunneling of electrons present in two-dimensional electron gas layer.

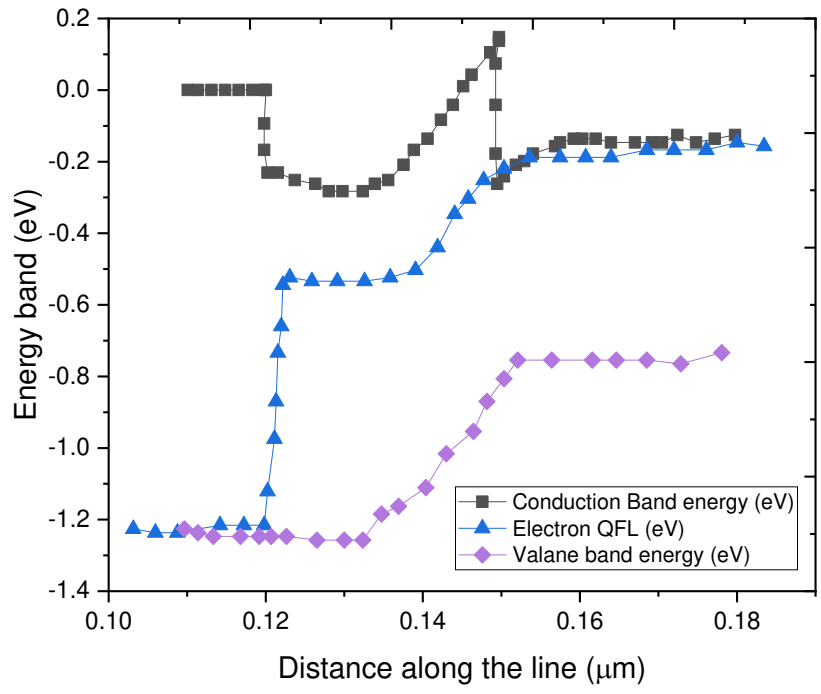

Fig.3. ON state Energy Band diagram of the AlGaN/GaN based high electron mobility transistor

According to energy band diagram observations there is almost same energy required in both the cases to move one electron from valance band to conduction band. So finally proposed device structure and ON state band diagram of the high electron mobility transistor has been drawn in above part and analysis has been done behind them.The conduction path was created by joining layer of $\mathrm{AlGaN} / \mathrm{GaN}$ in high electron mobility transistor structure causes positive net charges, which pull the conduction band towards downwards. The positive change density is now attracted by electrons and trap at potential well at triangular shaped. Moreover, the polarization shifted net negative charges which now dragged the conduction band upwards. The confined electron will create 2-DEG at the $\mathrm{GaN}$ channel junction. The expression for 2DEG at the GaN channel can be described as:

$$
\begin{gathered}
n_{s}=\left(\frac{\sigma_{\text {int }}}{q}\right)-\left(\frac{\varepsilon_{0} \varepsilon_{r}}{q^{2} t_{\text {AlGaN }}}\right)\left(q \varphi_{b}+E_{F}\right. \\
\left.+\Delta E_{C}\right)
\end{gathered}
$$

Where, $q$ is the electron change, $\sigma$ is the $\mathrm{AlGaN} / \mathrm{GaN}$ sheet charges at the interface, $E_{F}$ is the fermi energy level, $\varphi_{b}$ is the gate contact Schottky barrier height which can change the threshold voltage of the device. $\Delta E_{C}$ represent the energy bandgap between $\mathrm{AlGaN}$ and $\mathrm{GaN}$, where dielectric constant effects the breakdown field strength. Due to different value energy band gap of $\mathrm{AlGaN}$ and $\mathrm{GaN}$, lattice mismatches occur which can be compensate by its thickness. Table II represent different properties of high electron mobility transistor structure which are arose by hetero structures, which generates a potential well for electron as a channel for easy movement of electron and forms 2DEG. 
Table II: Different properties of high electron mobility transistor material

\begin{tabular}{|c|c|c|c|c|c|c|}
\hline $\begin{array}{c}\text { Material } \\
\text { Properties }\end{array}$ & Si & GaAs & GaN & AlN & InN & $\mathrm{SiC}$ \\
\hline $\begin{array}{c}\text { Energy } \\
\text { bandgap }(\mathrm{eV})\end{array}$ & 1.12 & 1.42 & 3.4 & 6.2 & 0.9 & 3.26 \\
\hline $\begin{array}{r}\text { Dielectric } \\
\text { Constant }\left(\varepsilon_{r}\right)\end{array}$ & 11.8 & 13.1 & 8.9 & 8.5 & 15.3 & - \\
\hline $\begin{array}{r}\text { Breakdown } \\
\text { Field }(\mathrm{Mv} / \mathrm{cm})\end{array}$ & 0.3 & 0.4 & 3.0 & 11 & low & 3 \\
\hline $\begin{array}{l}\text { Velocity } \\
(\mathrm{cm} / \mathrm{s})\end{array}$ & $1 \times 10^{7}$ & $2 \times 10^{7}$ & $2 \times 10^{7}$ & - & $2 \times 10^{8}$ & - \\
\hline $\begin{array}{l}\text { Electron } \\
\text { Mobility } \\
\left(\mathrm{cm}^{2} / \mathrm{Vs}\right)\end{array}$ & 1350 & 8500 & 440 & 300 & 250 & 720 \\
\hline $\begin{array}{c}\text { Lattice } \\
\text { constant }(\AA)\end{array}$ & 5.43 & 5.65 & 3.19 & 3.1 & 3.53 & 3.0 \\
\hline $\begin{array}{c}\text { Thermal } \\
\text { conductivity } \\
(\mathrm{W} / \mathrm{cm} \mathrm{k})\end{array}$ & 1.5 & 0.5 & 1.3 & - & - & 4.9 \\
\hline
\end{tabular}

\section{RESULTS AND DISCUSSION}

In order to describe the parameter variation effects on the characteristics of an AlGaN-GaN high electron mobility transistor, the most important characteristics are discussed below, and with the help of these characteristics we analyze the fundamental concepts behind them.

\section{A. Impacts on $I_{d-}-V_{G S}$ Characteristics}

Fig.4 shows the DC I-V characteristics of an $\mathrm{AlGaN} / \mathrm{GaN}$ high electron mobility transistor on $\mathrm{Al}_{2} \mathrm{O}_{3}$ substrate at various doping levels. The drain current is plotted with the gate gate voltages at the drain source voltage of $2 \mathrm{~V}$. It is seen that at low level doping i.e. from $10^{10} / \mathrm{cm}^{3}$ to $10^{12} / \mathrm{cm}^{3}$ the drain current remain constant and it start increasing when doping level rises from $10^{13} / \mathrm{cm}^{3}$. At the doping level of $10^{15} / \mathrm{cm}^{3}$ maximum drain current $\mathrm{I}_{\mathrm{d}}=22 \mathrm{~mA}$ is achieved.

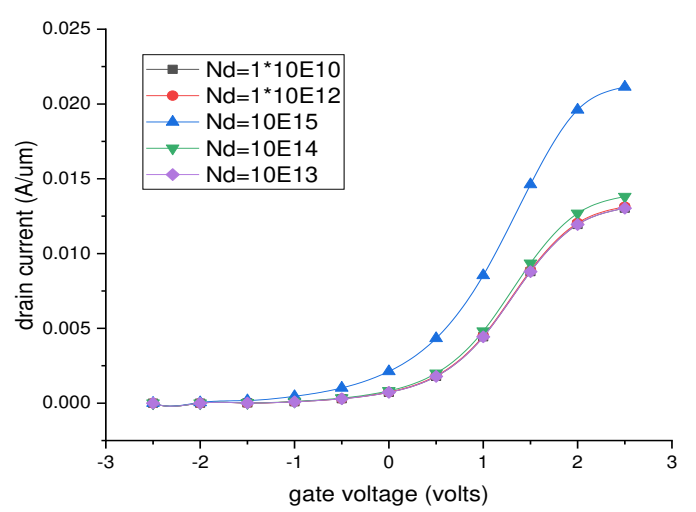

Figure 4 Id-Vgs characteristics for various doping concentration

The impact of gate work fuction (wf) on the drain current is plotted on the Fig.5. This graph is plotted keeping the constant doping level of $10^{15} / \mathrm{cm}^{3}$ and at the $\mathrm{Vds}=2 \mathrm{~V}$. It can be noticed that on increasing the gate work function from $4 \mathrm{eV}$ to $4.5 \mathrm{eV}$ initially the drain current increses by 2 orders but on futher increasing the work function the drain current starts decreasing. So, the maximum drain current is observed at gate work function of $4.5 \mathrm{eV}$.

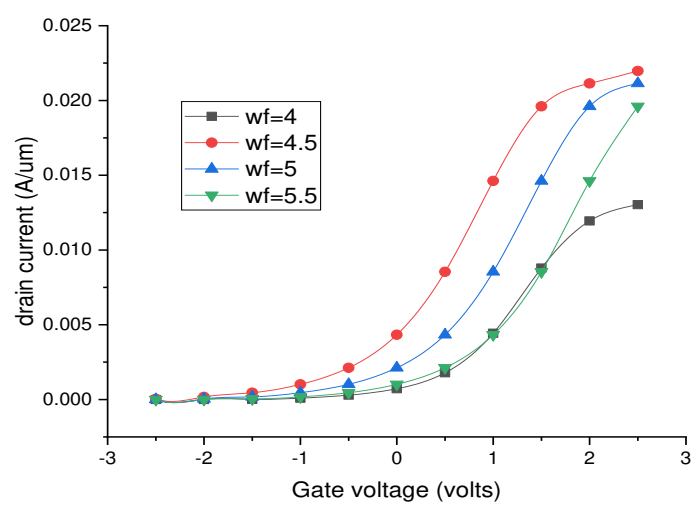

Figure 5 Id-Vgs characteristics for various gate work functions

According to the above characteristics, the threshold voltage is directly proportional to the doping of the device. Threshold voltage expression is given below.

$$
\begin{gathered}
V_{T H}=V_{F B}+2 \varphi_{F}+\gamma \sqrt{\varphi_{F}} \\
\gamma=\frac{\sqrt{2 q \varepsilon N}}{C_{o x}}
\end{gathered}
$$

Now third observation has been taken that is impacts of workfunction on I-V characteristics. Threshold voltage is again directly proportional to work-function of metal so the characteristics show slightly high threshold voltage compared to previous results.

$$
V_{t h}=\text { Ideal threshold volt. }+V_{F B}
$$

Where, $V_{F B}$ is the flat band voltage.

$$
V_{F B}=\phi_{m s}-\frac{Q_{f c}}{C_{o x}}
$$

The variation of the drain current with different channel length are plotted in Fig. 6 and Fig. 7 at high bulk doping and low bulk dping respectively. The simulation is carried out at the gate wok function of 4. $\mathrm{eV}$ and $\mathrm{Vds}=2 \mathrm{~V}$. It is noticed that at the high doping level the drain current decreases on increasing the channel length while at the low doping level the variation is less as compare to the high doping level. Threshold voltage is very small compared to the previous one because the charge in the oxide increases, that is mentioned in above equations (9) \& (10). It is also observed that the drain current at the low level doping is $78 \%$ lower than the drain current at high doping level. 


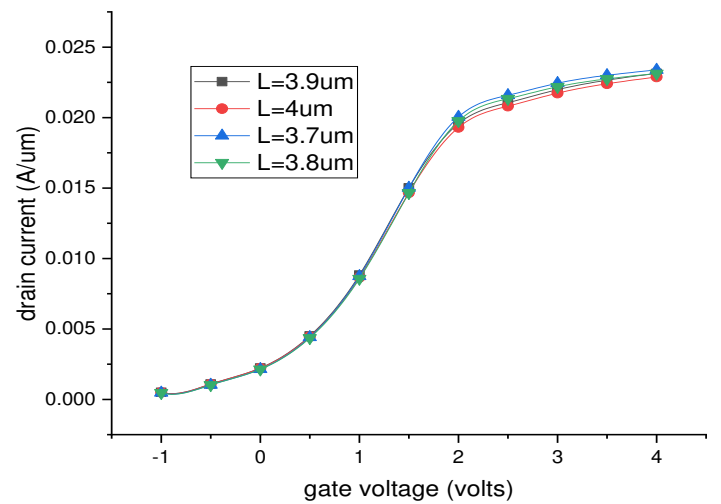

Figure 6 Id-Vgs characteristics for various channel length at high doping concentration

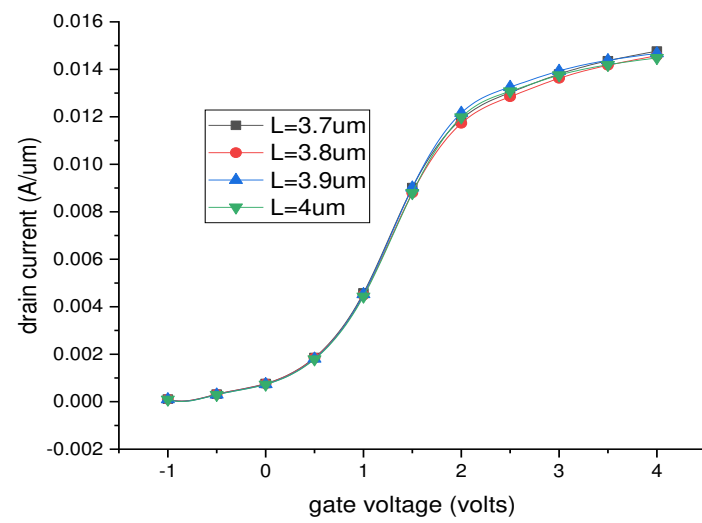

Figure 7 Id-Vgs characteristics for various channel length at low doping concentration

\section{B. Impacts on $g_{m}-V_{G S}$ Characteristics}

The trans-conductance is defined as change in drain current with respect to gate to source voltage at constant $V_{d s}$.

$$
g m=\frac{\partial I_{D}}{\partial V_{G S}}
$$

Fig.8 shows the characteristics of $g_{m}$ vs. $V_{g s}$ at the different doping concentarion of the bulk. Transconductance shows maximum at value $\mathrm{V}_{\mathrm{gs}}=1.5 \mathrm{~V}$ and at the doping level of $1 \times 10^{16} / \mathrm{cm}^{3}$. Initially the the transconductnce increses with increase in the doping but eventually it start decreasing when the doing level reaches to $1 \times 10^{18} / \mathrm{cm}^{3}$.

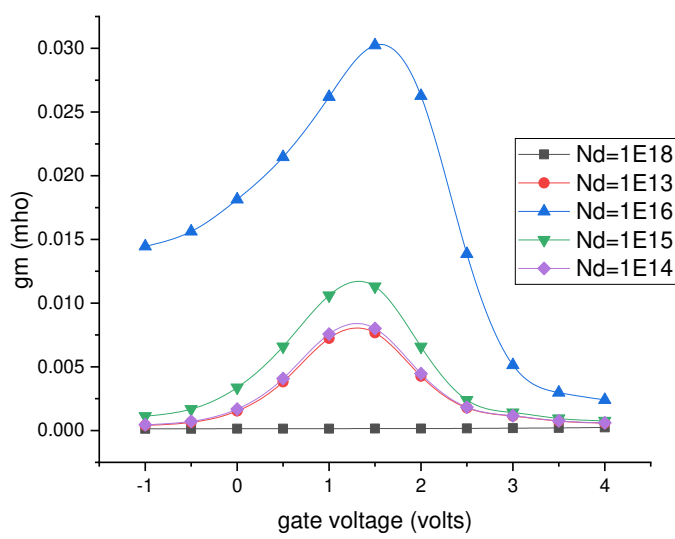

Figure $8 g_{m}$ vs Gate voltage characteristics at different doping level

For RF applications $g_{m}$ should be maximum because the cutoff frequency of the device should be large and $g_{m}$ is directly proportional to $\mathrm{f}_{\mathrm{T}}$. Now the trans-conductance depends upon various parameters so the mathematical equation of the transconductance is given below-

$$
\begin{gathered}
g_{m}=\mu_{n} C_{o x} \frac{W}{L}\left(V_{g s}-V_{t h}\right) \\
g_{m}=\sqrt{2 \mu_{n} C_{o x} \frac{W}{L} I_{D}}
\end{gathered}
$$

Comparison between two $\mathrm{g}_{\mathrm{m}}$ characteristics clearly shows the effect of doping concentration on the trans-conductance and it is observed that trans-conductance is inversely proportional to the doping concentration. We have observed that impact ionization increases with the decrease in the doping concentration and vice versa.

The effect of metal gate work function on the transconductance is plotted in Fig. 9. Which shows $g_{m}$ is maximum at $\mathrm{V}_{\mathrm{gs}}=1 \mathrm{~V}$ for the work function of $4.5 \mathrm{eV}$. the maximum value of the $g_{m}$ is obtained as $11 \mathrm{~m}$ mho. Equation (12) already mentions that $\mathrm{g}_{\mathrm{m}}$ is linear and inversely dependent on threshold voltage so it is ultimately inversely dependent on gate work-function.

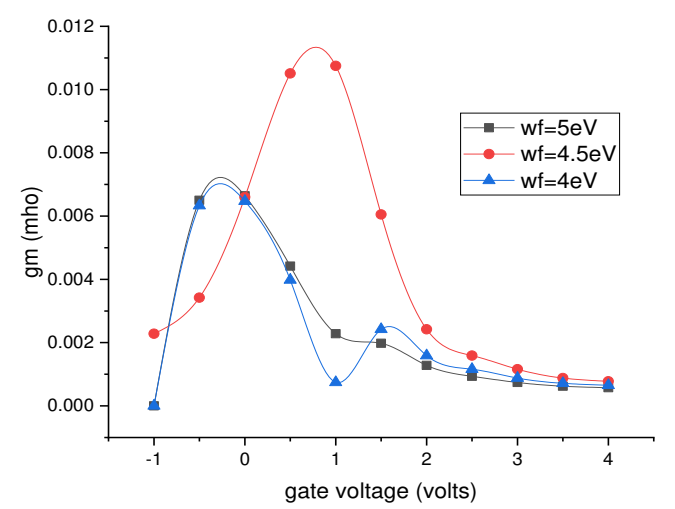

Figure 9. gm vs Gate voltage at different gate material work-function

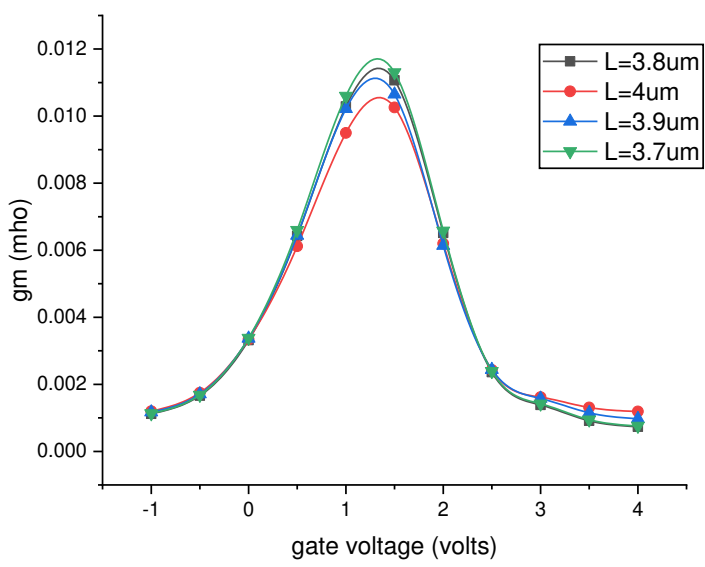

Figure 10. $g_{m}-V_{g s}$ characteristics for various channel length 
The variation of the transconductance with the channel length is plotted in Fig. 10. It is seen that on increasing the channel length the transconductance decreases because the drain current reduces with the increase in the channel length. If the channel length increases the drain current decreases because the number of electrons or electron density decreases so if the drain current gives approximate linear characteristic then the transconductance will be constant or saturate according to equation (11).

\section{Impacts on Gate Capacitance}

For RF applications, the gate capacitances; gate to source capacitance $\mathrm{Cgs}$ and gate to drain capacitance Cgd plays a major role while determining the device performance. gate voltage versus gate capacitance $\mathrm{Cgs}$ and $\mathrm{Cgd}$ characteristics are shown in Figure 11(a) and Fig. 11 (b) respectively at different doping variations. It is seen that initially there is little variation on the Cgs and Cgd with the doping but on further increasing the doping level in th bulk both source and drain capacitance increases since the number of chrge carrier increases. At the higher gate voltage its value varies in the range of $1.5 \mathrm{pF}$ to 2 $\mathrm{pF}$.
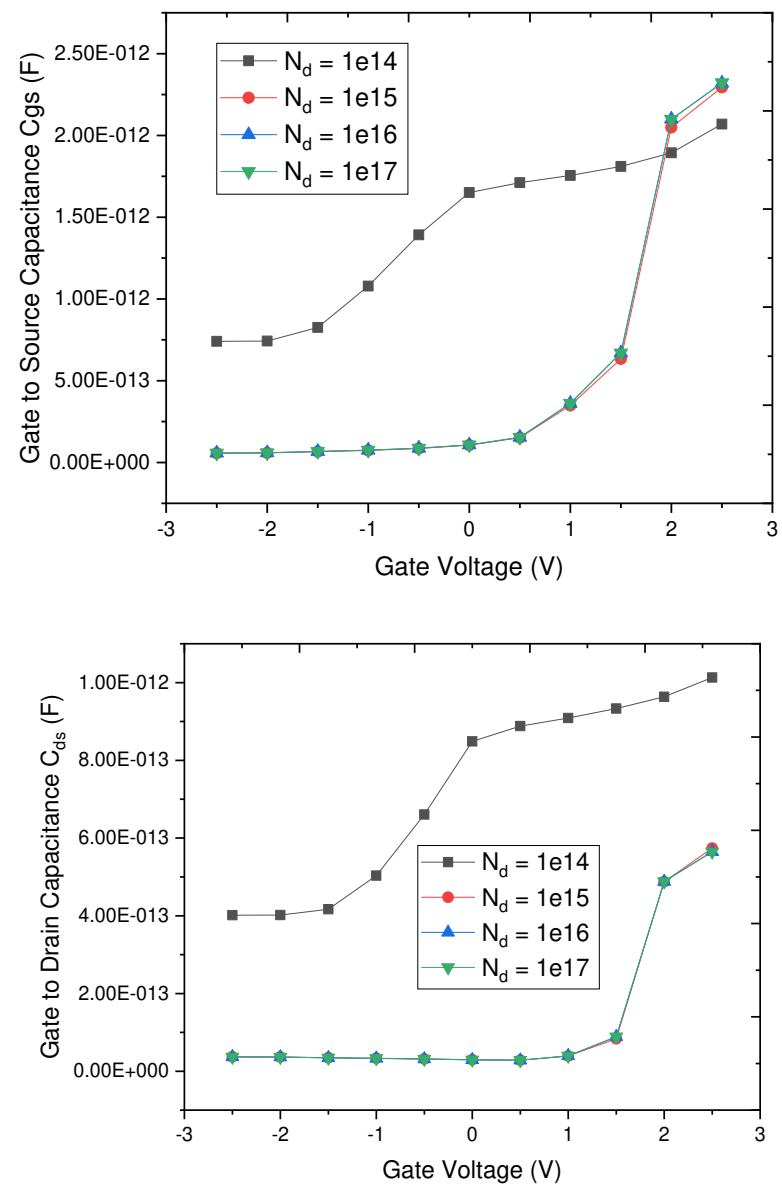

Figure 11 (a) $\mathrm{C}_{g s}-\mathrm{V}_{\mathrm{gs}}$, and (b) Cgd-Vgs Characteristics for various doping levels

Fig. 12 shows the variation of gate capacitance with the applied gate bias for different channel lengths. Fig. 12(a) is drawn for gate to source capacitance while Fig. 12 (b) is drawn for gate to drain capacitance. It is noticed that on increasing the channel length both capacitances $\mathrm{Cgs}$ and $\mathrm{Cgd}$ increase with the gate voltage. The obtaine values of the gate capacitance lies in the range of $0.2 \mathrm{pF}$ to $0.8 \mathrm{pF}$.
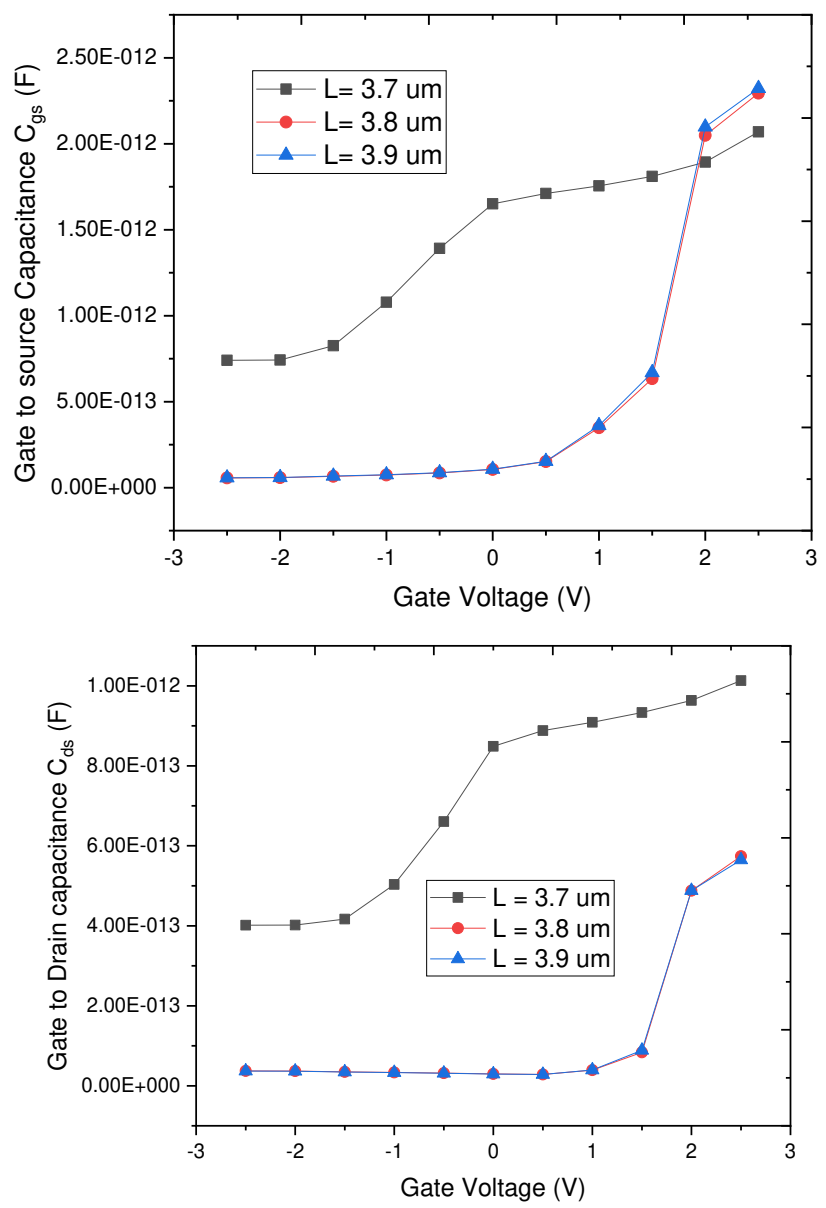

Fig. 12 Variation of (a) gate to source capciatnce Cgs and (b) gate to drain capacitance Cgd for different channel length

The impct of work function on the variation of the gate to source capciatnce $\mathrm{Cgs}$ and gate to drain capacitance $\mathrm{Cgd}$ is plotted in the Fig 13 (a) and Fig. 13 (b) respectively. It is observed that the both capaciatnces increase when the metal work function rises and their values lie in the range of $0.2 \mathrm{pF}$ to $1 \mathrm{pF}$.

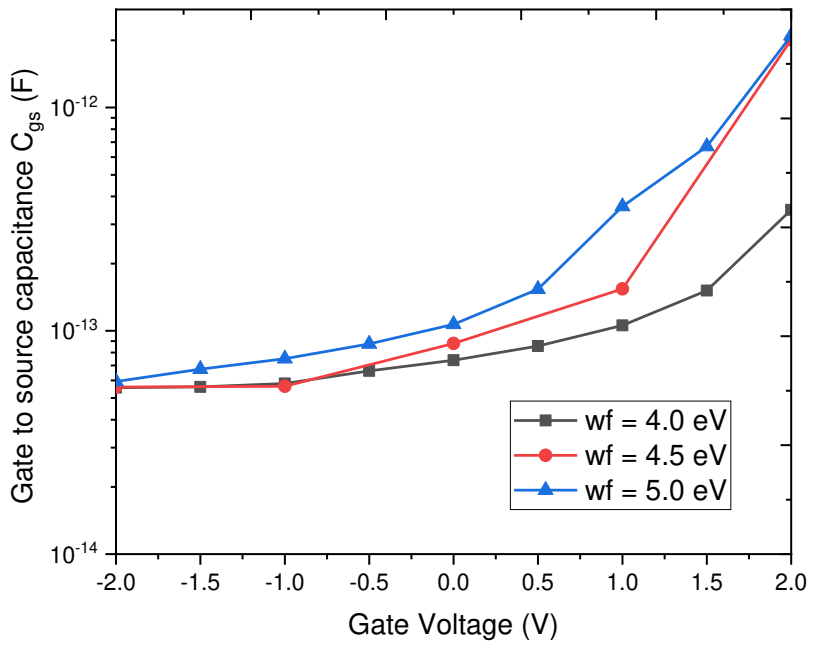




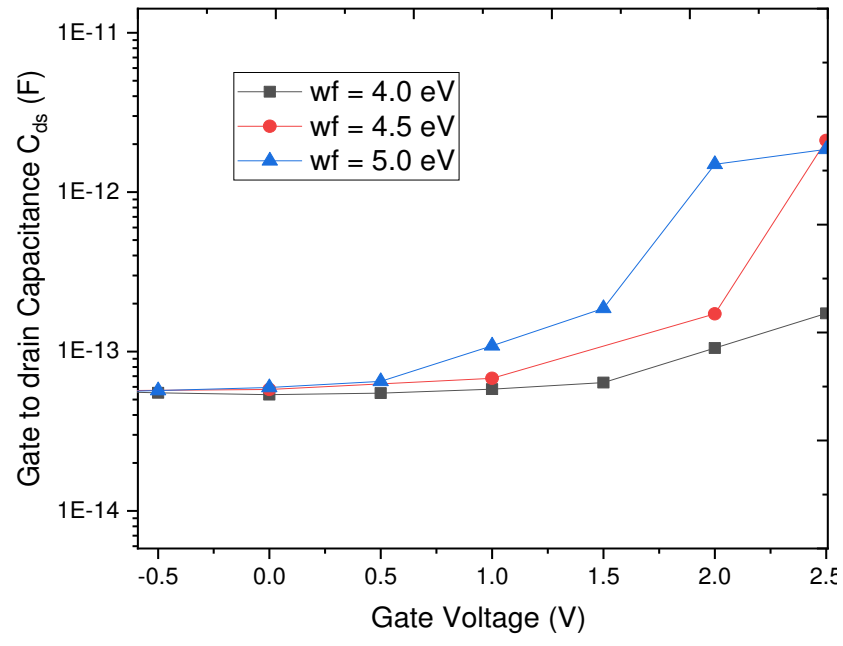

Fig. 13 Variation of (a) gate to source capciatnce Cgs and (b) gate to drain capacitance $\mathrm{Cgd}$ for different metal gate work function

The cut-off frequency expression is given as-

$$
f_{T}=\frac{g_{m}}{2 \pi C_{g}}
$$

The gain falls at high frequency and low frequency so there is a frequency range where the gain of the device is maximum. According to equation (14) the cut-off frequency depends on two parameters so $\mathrm{g}_{\mathrm{m}}$ is directly proportional and overall gate capacitance is inversely proportional to cut-off frequency.

Therefore, $\mathrm{C}_{\mathrm{g}}$ should be less and $\mathrm{g}_{\mathrm{m}}$ should be high to acquire high cut-off frequency range. If we observe the characteristic, then doping plays a major role in capacitance so in one hand, the doping of device should be less but for good conduction and for high gain the doping is required.

\section{Conclusion}

This paper presents design and comparative performance analysis of the high electron mobility transistor on silicon substrate for RF applications. The variation in the performance parameters drain current, transconductance, and total gate capacinatce is analyzed by varying the various design parameters like doping level, channel length and work-function of gate material. In the proposed device a good performance with maximum $\mathrm{g}_{\mathrm{m}}=13$ milli-mho, minimum gate capacitance $\mathrm{C}_{\mathrm{g}}=0.5 \mathrm{pf}$ is achieved. $\mathrm{V}_{\text {th }}$ is varied between $-0.25 \mathrm{~V}$ to $0.25 \mathrm{~V}$ at various status of device parameters. One characteristic is basic device parameter and other two RF characteristics are investigated.

Data Availability: Not applicable.

\section{Author contributions:}

All authors have equally participated in the preparing of the manuscript during implementation of ideas, findings results, and writing of the manuscript.

\section{Declarations}

Funding statement: The author(s) received no financial support for the research, authorship, and/or publication of this article.

Availability of data and material: Not applicable

Compliance with ethical standards: all procedures performed in studies involving human participants were in accordance with the ethical standards.

\section{Consent to participate: Not applicable}

Consent for Publication: The Author transfers his copyrights to the publisher.

Conflict of Interest: The authors declare that there are no conflicts of interest.

Acknowledgement: Not Applicable

\section{REFERENCES}

[1] Egawa, T. "Development of next generation devices amidst global competition due to their huge market potential." Ultimate in Vacuum ULVAC 63 (2012): 18-21.

[2] Hoefflinger, Bernd. "ITRS: The international technology roadmap for semiconductors." In Chips 2020, pp. 161-174. Springer, Berlin, Heidelberg, 2011

[3] Ma, Ke, Ning He, Marco Liserre, and Frede Blaabjerg. "Frequencydomain thermal modeling and characterization of power semiconductor devices." IEEE Transactions on Power Electronics 31, no. 10 (2015): 7183-7193.

[4] Baliga, B. Jayant. "Trends in power semiconductor devices." IEEE Transactions on electron Devices 43, no. 10 (1996): 1717-1731.

[5] Shenai, Krishna. "The figure of merit of a semiconductor power electronics switch." IEEE Transactions on Electron Devices 65, no. 10 (2018): 4216-4224.

[6] Boles, T., 2018, February. GaN-on-Silicon-Present capabilities and future directions. In AIP Conference Proceedings (Vol. 1934, No. 1, p. 020001). AIP Publishing LLC.

[7] Kuzmik, J., Javorka, R., Alam, A., Marso, M., Heuken, M. and Kordos, P., 2002. Determination of channel temperature in AlGaN/GaN HEMTs grown on sapphire and silicon substrates using DC characterization method. IEEE transactions on electron devices, 49(8), pp.1496-1498.

[8] Tsung-Hsing Yu and Kevin F. Brennan - Theoretical Study of a GaNAlGaN High Electron Mobility Transistor Including a Nonlinear Polarization Model, IEEE TRANSACTIONS ON ELECTRON DEVICES, VOL. 50, NO. 2, FEBRUARY 2003.

[9] Canberk Dundar, Dogacan Kara, and Nazli Donmezer, Member, IEEE The Effects of Gate-Connected Field Plates on Hotspot Temperatures of AlGaN/GaN HEMTs on IEEE TRANSACTIONS ON ELECTRON DEVICES.

[10] GaN-Based HEMTs for High Voltage Operation Design, Technology and Characterization Vorgelegt von B. Sc. Eng, M. Sc. Eng. Eldad BahatTreidel aus Jerusalem, Israel- Vorsitzender: Prof. Dr. Wolfgang Heinrich Berichter: Prof. Dr. Günther Tränkle Berichter: Prof. Dr. Michael Uren Tag der wissenschaftlichen Aussprache: 30.3.2012.

[11] Sen Huang, Xinyu Liu, Xinhua Wang, Xuanwu Kang, Jinhan Zhang, Qilong Bao, Ke Wei, Yingkui Zheng, Chao Zhao, Hongwei Gao, Qian Sun, Zhaofu Zhang, and Kevin J. Chen, Fellow, IEEE - High Uniformity Normally-OFF GaN MISHEMTs Fabricated on Ultra-Thin-Barrier AlGaN/GaN Heterostructure, IEEE Electron Device Letters, 0741-3106 (c) 2016 IEEE.

[12] Sheikh Aamir Ahsan, Graduate Student Member, IEEE, Sudip Ghosh, Khushboo Sharma, Avirup Dasgupta, Graduate Student Member, IEEE, Sourabh Khandelwal, Member, IEEE, and Yogesh Singh Chauhan, Senior Member, IEEE - Capacitance Modeling in Dual Field-Plate Power GaN HEMT for Accurate Switching Behavior, November 27, 2015

[13] Giuseppe Grecoa, Ferdinando Iucolanob, Fabrizio Roccaforte - Review of technology for normally-off HEMTs with $\mathrm{p}-\mathrm{GaN}$ gate, Consiglio 
Nazionale delle Ricerche - Istituto per la Microelettronica e Microsistemi (CNR-IMM), Strada VIII, n. 5 - Zona Industriale, 95121 Catania, Italy b STMicroelectronics, Stradale Primosole 50, 95121 Catania, Italy.

[14] TYLER J. FLACK, 1,2 BEJOY N. PUSHPAKARAN,1 and STEPHEN B. BAYNE - GaN Technology for Power Electronic Applications: A Review, Journal of ELECTRONIC MATERIALS DOI: 10.1007/s11664016-4435-3 2016 The Minerals, Metals \& Materials Society.

[15] A.S.A. Fletcher, D. Nirmal, A survey of Gallium Nitrate HEMT for RF and high power applications, Superlattices and Microstructures (2017), doi: 10.1016/j.spmi.2017.05.042.

[16] Finella Lee, Liang-Yu Su, Chih-Hao Wang, Yuh-Renn Wu, and Jianjang Huang, Senior Member, IEEE - Impact of Gate Metal on the Performance of p-GaN/AlGaN/GaN High Electron Mobility Transistors, IEEE ELECTRON DEVICE LETTERS, VOL. 36, NO. 3, MARCH 2015.

[17] Tallarico, Andrea Natale, Steve Stoffels, Niels Posthuma, Stefaan Decoutere, Enrico Sangiorgi, and Claudio Fiegna. "Threshold voltage instability in GaN HEMTs with p-type gate: Mg doping compensation." IEEE Electron Device Letters 40, no. 4 (2019): 518-521.

[18] Jurkovic, Michal, Dagmar Gregusova, Vassil Palankovski, Štefan Hascik, Michal Blaho, Karol Cico, Karol Frohlich, Jean-Francois Carlin, Nicolas Grandjean, and Jan Kuzmik. "Schottky-barrier normally off GaN/InAlN/AIN/GaN HEMT with selectively etched access region." IEEE electron device letters 34, no. 3 (2013): 432-434.

[19] Muhea, Wondwosen Eshetu, Fetene Mulugeta Yigletu, Roger CabreRodon, and Benjamin Iniguez. "Analytical model for Schottky barrier height and threshold voltage of AlGaN/GaN HEMTs with piezoelectric effect." IEEE Transactions on Electron Devices 65, no. 3 (2018): 901907.

[20] Ao, Jin-Ping, Daigo Kikuta, Naotaka Kubota, Yoshiki Naoi, and Yasuo Ohno. "Copper gate $\mathrm{AlGaN} / \mathrm{GaN}$ HEMT with low gate leakage current." IEEE Electron Device Letters 24, no. 8 (2003): 500-502.

[21] TCAD Atlas User's Manual, SILVACO $r$ interna- tional (2018)

[22] Khandelwal, Sourabh, Nitin Goyal, and Tor A. Fjeldly. "A physics-based analytical model for 2DEG charge density in AlGaN/GaN HEMT devices." IEEE Transactions on Electron Devices 58, no. 10 (2011): 36223625.

[23] Jebalin, Binola K., A. Shobha Rekh, P. Prajoon, D. Godwinraj, N. Mohan Kumar, and D. Nirmal. "Unique model of polarization engineered AlGaN/GaN based HEMTs for high power applications." Superlattices and Microstructures 78 (2015): 210-223.

[24] Jain, S. C., M. Willander, J. Narayan, and R. Van Overstraeten. "IIInitrides: Growth, characterization, and properties." Journal of Applied Physics 87, no. 3 (2000): 965-1006.

[25] Feng, Z. H., Yu Gang Zhou, S. J. Cai, and Kei May Lau. "Enhanced thermal stability of the two-dimensional electron gas in Ga N Al Ga N Ga N heterostructures by Si $3 \mathrm{~N} 4$ surface-passivation-induced strain solidification." Applied physics letters 85, no. 22 (2004): 5248-5250.

[26] Javorka, Peter. "Fabrication and characterization of AlGaN/GaN high electron mobility transistors." Bibliothek der RWTH Aachen (2004).

[27] Bisi, Davide, et al. "Trapping mechanisms in GaN-based MIS-HEMTs grown on silicon substrate." physica status solidi (a) 212.5 (2015): 11221129 .

[28] Meneghesso, Gaudenzio, et al. "Anomalous kink effect in GaN high electron mobility transistors." IEEE Electron Device Letters 30.2 (2008): 100-102.

[29] L. Jimenez, "Recent advances on the understanding of the physics of failure of GaN on SiC FET technology", Proc. IEEE Int. Reliability Physics Symp., Phoenix, USA, pp.429- 435, 2008. 